CU-TP-1106

\title{
De Sitter Space With Finitely Many States: A Toy Story
}

\author{
MAUlik PARIKH ${ }^{\mathrm{a}}$ AND ERIK Verlinde \\ ${ }^{a}$ Department of Physics, Columbia University, New York, NY 10027 \\ ${ }^{b}$ Physics Department, University of Amsterdam, The Netherlands
}

\begin{abstract}
The finite entropy of de Sitter space suggests that in a theory of quantum gravity there are only finitely many states. It has been argued that in this case there is no action of the de Sitter group consistent with unitarity. In this note we propose a way out of this if we give up the requirement of having a hermitian Hamiltonian. We argue that some of the generators of the de Sitter group act in a novel way, namely by mixing in- and out-states. In this way it is possible to have a unitary S-matrix that is finite-dimensional and, moreover, de Sitter-invariant. Using Dirac spinors, we construct a simple toy model that exhibits these features. ${ }^{\mathrm{c}}$
\end{abstract}

\section{Introduction}

In AdS/CFT, the $O(2,4)$ isometry group of anti-de Sitter space is reinterpreted as conformal symmetry of the dual theory. For de Sitter space, we do not yet possess the holographically dual theory; nevertheless, we would expect the $O(1, d)$ isometry group of de Sitter space to be a symmetry of that theory. But, unlike AdS, de Sitter space also has horizons, with an associated entropy. There are differing interpretations of the entropy but one view, which we shall explore here, is that it indicates that the dual theory has only a finite number of states 12 .

However, there is a well-known theorem that says that there are no nontrivial finite-dimensional unitary representations of a noncompact group. Consequently,

amkp@phys.columbia.edu

berikv@science.uva.nl

${ }^{\mathrm{c}}$ Based in part on a talk presented by one of us (M. P.) at the Xth Marcel Grossmann Meeting, held in Rio de Janeiro, July 20-26, 2003. 
the Fock space of the dual theory can be either finite-dimensional or a unitary representation of the de Sitter group, but not both. There have thus been claims

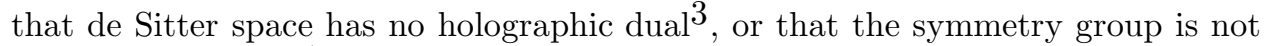
the de Sitter group 415 .

Here we will consider the possibility that the dual to de Sitter space is indeed defined by a finite-dimensional Fock space, so that the entropy is finite. The finiteness of the Fock space requires that particles in the dual theory obey Fermi-Dirac statistics. Hence we shall construct a spinor dual to de Sitter space. The finitely many Fock states describing a static patch of de Sitter space do not form representations of the de Sitter group but only of the (compact) rotation subgroup. Global de Sitter space is described by two copies of the Fock space. After antipodal identification, these become the space of initial and final states. Some of the de Sitter generators then mix the in- and out-states i.e. bras with kets. The main idea is that there are certain pairings of bras and kets that are nevertheless de Sitter-invariant. These can be used to construct a de Sitter-invariant S-matrix so, in that sense, the isometry group of de Sitter space indeed appears as a symmetry of the dual theory. In this paper, we will illustrate the reconciliation of symmetry and entropy with a toy model based on Dirac spinors; a more refined construction involving fuzzy spheres will appear in a companion paper ${ }^{6}$.

\section{Fock States and Tensor Product States}

De Sitter space is conveniently thought of as a timelike hyperboloid embedded in Minkowski space:

$$
-X^{0^{2}}+X^{1^{2}} \cdots+X^{d^{2}}=+R^{2}
$$

where $X^{I}$ are Cartesian embedding coordinates. In this form, the $O(1, d)$ isometry group of $d$-dimensional de Sitter space is manifest: it is the Lorentz group in $d+1$ dimensions. Now, consider a geodesic observer in de Sitter space. Without loss of generality, let the observer be at the north pole, which we take to be in the positive $X^{d}$ direction. The Lorentz generators that leave the observer's worldline invariant are the rotations about the axis connecting the poles as well as the boost in the $X^{d}$ direction. Together these form the observer's little group, $O(d-1) \times R$. Note that since the rotation group $O(d-1)$ is compact, it admits finite-dimensional unitary representations.

Now, since the states counted by the entropy are associated with a horizon, we should take the Hilbert space to form a representation of the group that keeps the horizon fixed. This is just the observer's little group. Hence we take the one-particle Hilbert space, $h_{I}$, to be a finite-dimensional unitary representation of $O(d-1)$. (It is, however, a nonunitary representation of $O(d-1) \times R$, as we will see.) We denote the corresponding multi-particle Hilbert space, or Fock space, by $H_{I}$. In order to obtain a finite-dimensional Fock space, the particles need to obey Fermi-Dirac statistics. Similarly, the states accessible to the antipodal observer at the south pole 
belong to a finite-dimensional Fock space, $H_{I I}$, constructed out of a one-particle Hilbert space, $h_{I I}$, that is isomorphic to that of the observer at the north pole. The state describing global de Sitter space is a tensor product of states in the Fock spaces of antipodal pairs of observers. A typical basis state of the dual to global de Sitter space is therefore

$$
|m\rangle_{I} \otimes|n\rangle_{I I}
$$

where $m$ and $n$ are quantum numbers of $O(d-1)$. The general state is

$$
|\Psi\rangle=\sum_{m, n} C_{m n}|m\rangle_{I} \otimes|n\rangle_{I I}
$$

Mixed states that cannot be written in the form $|\psi\rangle_{I} \otimes|\phi\rangle_{I I}$ are those that correlate the antipodal pair of observers. The state corresponding to a particle beyond the future horizons of both the observers would be an example of a mixed state.

The tensor product states live in the direct product space $H_{I} \otimes H_{I I}$. This implies that the one-particle Hilbert space for global de Sitter space is a direct sum of the two observers' Hilbert spaces. One can check this by counting the number of states: if there are $n$ states in an observer's one-particle Hilbert space, then there are $2^{n}$ states in the Fock space, assuming Fermi-Dirac statistics, and hence $2^{n} \times 2^{n}=2^{2 n}$ states in the global (tensor product) Fock space. So the global Hilbert space, $h$, has $2 n$ states, indicating that it is a direct sum of the two Hilbert spaces:

$$
h=h_{I} \oplus h_{I I} .
$$

Consider now a general de Sitter transformation, $M$. The Fock spaces $H_{I}$ and $H_{I I}$ are $O(d-1)$-invariant by construction, but they are not de Sitter-invariant. This is because a de Sitter transformation that is not in $O(d-1) \times R$ can take a particle out of an observer's horizon into the antipodal observer's horizon. So on the global one-particle Hilbert space, $h, M$ acts as $M: h \rightarrow h$ i.e. as

$$
M: h_{I} \oplus h_{I I} \rightarrow h_{I} \oplus h_{I I} .
$$

More generally, on the space of tensor products of Fock states, $M$ acts as

$$
M: H_{I} \otimes H_{I I} \rightarrow H_{I} \otimes H_{I I} .
$$

Of course, since the individual Fock spaces are finite-dimensional, the tensor product space is also finite-dimensional. Hence it also falls afoul of the theorem forbidding nontrivial unitary finite-dimensional representations of the de Sitter group. But we can find certain special tensor product states that transform in a trivial representation of the de Sitter group. That is, we will look for those tensor product states, $|S\rangle$, that are invariant under the de Sitter group, $M|S\rangle=0$. The Fock space accessible to an individual observer nevertheless has a finite number of states, so the entropy is finite. We will argue that if we identify antipodal points in de Sitter space, the tensor product states become processes; the special tensor product states, $|S\rangle$, then correspond to de Sitter-invariant processes. 


\section{A Toy Model for de Sitter Space}

We now construct a toy model based on Dirac spinors. This is perhaps the simplest dual to de Sitter space. There are a couple of motivations for considering spinors. Because of spin-statistics, the Fock space of fermions can be finite-dimensional. For bosons, the Fock space would be infinite-dimensional even for a finite-dimensional Hilbert space as there would be no restriction on the number of bosons. Another reason for considering spinor representations is that the number of components of a Dirac spinor doubles when the dimension is increased by two. So for Dirac representations, $R$, we have

$$
\operatorname{dim} O(d-1)_{R}=\frac{1}{2} \operatorname{dim} O(1, d)_{R} .
$$

This allows us to write a global Hilbert state, which transforms under $O(1, d)$, as a direct sum of two states that transform under $O(d-1)$, in accordance with (5).

The gamma matrices obey the Clifford algebra $\left\{\Gamma_{I}, \Gamma_{J}\right\}=2 \eta_{I J}$, where $I, J=$ $0 \ldots d$. A convenient representation is

$$
\Gamma_{i}=\sigma_{3} \otimes \gamma_{i}=\left(\begin{array}{cc}
\gamma_{i} & 0 \\
0 & -\gamma_{i}
\end{array}\right), \Gamma_{0}=i \sigma_{2} \otimes 1=\left(\begin{array}{rr}
0 & 1 \\
-1 & 0
\end{array}\right), \Gamma_{d}=\sigma_{1} \otimes 1=\left(\begin{array}{ll}
0 & 1 \\
1 & 0
\end{array}\right)
$$

where $\gamma_{i}$ are the gamma matrices for the Clifford algebra $\left\{\gamma_{i}, \gamma_{j}\right\}=2 \delta_{i j}$, with $i, j$ running from 1 to $d-1$. The top half of the $\Gamma$ matrices act on a spinor state in $h_{I}$, with the bottom half acting on $h_{I I}$.

The de Sitter generators can then be written as

$$
M_{I J}=-\frac{i}{4}\left[\Gamma_{I}, \Gamma_{J}\right]
$$

It is convenient to express the de Sitter generators in terms of indices $i, j$ that run from 1 to $d-1$ :

$$
J_{i j} \equiv M_{i j} \quad P_{i} \equiv M_{d i} \quad K_{i} \equiv M_{0 i} \quad H \equiv M_{0 d} .
$$

Here $J_{i j}$ generate the rotation group $S O(d-1), P_{i}$ are momentum operators, $K_{i}$ are the boosts, and $H$ is the generator of time translations. To be specific, consider four-dimensional de Sitter space. The four-dimensional de Sitter group is $O(1,4)$ and the observer's little group is $O(3)$. Then, in the above representation, the de Sitter generators are

$$
J_{i}=\frac{1}{2}\left(\begin{array}{cc}
\sigma_{i} & 0 \\
0 & \sigma_{i}
\end{array}\right), P_{i}=\frac{i}{2}\left(\begin{array}{cc}
0 & \sigma_{i} \\
-\sigma_{i} & 0
\end{array}\right), K_{i}=\frac{i}{2}\left(\begin{array}{cc}
0 & \sigma_{i} \\
\sigma_{i} & 0
\end{array}\right), H=\frac{i}{2}\left(\begin{array}{cc}
-1 & 0 \\
0 & 1
\end{array}\right)
$$

where $\sigma_{i}$ are the Pauli matrices and $J_{i}=\frac{1}{2} \epsilon_{i j k} J_{j k}$. Notice that $P_{i}$ and $K_{i}$ are off-diagonal, indicating that they do not act within the Hilbert space of a single observer. In this representation, $J_{i}$ and $P_{i}$ are hermitian whereas $K_{i}$ and $H$ are not. That the Hamiltonian, $H$, is anti-hermitian may seem odd, but it may just indicate that any excitation of empty de Sitter space decays to empty de Sitter space. It 
should also be stressed that $H$ acts on states in quantum gravity which presumably live at the boundary; there is no obvious connection between the eigenvalues of $H$ and the energy measured perturbatively by an observer in the bulk.

The one-particle Hilbert space, $h_{I}$, consists of just two states: the twocomponent spinors, $|\uparrow\rangle$ and $|\downarrow\rangle$. The antipodal observer has an isomorphic Hilbert space, $h_{I I}$, and the above $4 \times 4$ matrices act on the direct sum of these two Hilbert spaces. The Fock space consists of the four states $|0\rangle,|\uparrow\rangle,|\downarrow\rangle$, and $|\uparrow \downarrow\rangle$. These four states can be labeled by their transformation properties under $O(3)$. Obviously, they belong to the $\mathbf{1}$, the $\mathbf{2}$, and the $\mathbf{1}$ representations, respectively.

The antipodal observer has an isomorphic Fock space. The tensor product states therefore transform under the direct product of the respective representations. Taking the direct product of the $\mathbf{1}$, the $\mathbf{2}$, and the $\mathbf{1}$ with themselves we find that the 16 tensor product states belong to

$$
\mathbf{1} \oplus \mathbf{2} \oplus \mathbf{1} \oplus \mathbf{2} \oplus \mathbf{3} \oplus \mathbf{1} \oplus \mathbf{2} \oplus \mathbf{1} \oplus \mathbf{2} \oplus \mathbf{1} .
$$

These are $O(3)$ labels. We are interested in how the tensor product states transform not just under rotations, but under general de Sitter transformations. We can group the above $O(3)$ representations into $O(1,4)$ representations:

$$
\mathbf{1} \oplus \mathbf{4} \oplus \mathbf{5} \oplus \mathbf{1} \oplus \mathbf{4} \oplus \mathbf{1} .
$$

For example, the states $|0\rangle_{I} \otimes|\uparrow\rangle_{I I},|0\rangle_{I} \otimes|\downarrow\rangle_{I I},|\uparrow\rangle_{I} \otimes|0\rangle_{I I}$, and $|\downarrow\rangle \otimes|0\rangle_{I I}$ together combine to form a $\mathbf{4}$, which is a spinor of $O(1,4)$. The $\mathbf{5}$ is a vector of $O(1,4)$.

Notice, in particular, that there are three singlet states. These are tensor product states that are invariant under the action of the de Sitter group. The three invariant states are

$$
|0\rangle \otimes|0\rangle,|\uparrow \downarrow\rangle \otimes|\uparrow \downarrow\rangle,(|\uparrow\rangle \otimes|\downarrow\rangle-|\downarrow\rangle \otimes|\uparrow\rangle)
$$

\section{A De Sitter-Invariant S-Matrix}

So far we have considered the Fock spaces $H_{I}$ and $H_{I I}$ as being isomorphic but otherwise independent spaces. De Sitter-invariance is implemented through choosing particular tensor products of states that are invariant under the full de Sitter group. This group-theoretic construction is correct as far as it goes but we can make it more compelling from a physical standpoint if we consider antipodally-identified or "elliptic" de Sitter space 789. This consists of identifying events that are related by the $\mathbb{Z}_{2}$ antipodal map

$$
X^{I} \rightarrow-X^{I}
$$

where $X^{I}$ are the Cartesian embedding coordinates, together with charge conjugation, $\mathrm{C}^{8}$.

Antipodal identification identifies points at time $X^{0}$ with those at $-X^{0}$. As a result, elliptic de Sitter space is not time-orientable; there is no globally-consistent way to distinguish future light cones from past light cones. In particular, the arrow 
of time in the antipodal observer's causal patch points in the opposite sense. This suggests that if we think of $H_{I}$ as the space of initial kets, then the antipodal Fock space should be regarded as the space of final bras 810 . The tensor product states $|m\rangle_{I} \otimes|n\rangle_{I I}$ can now be thought of as a physical process $|m\rangle_{\text {in }} \rightarrow\left|n^{\prime}\right\rangle_{\text {out }}$. Here the ' indicates that the out-state $\left|n^{\prime}\right\rangle_{\text {out }}$ is actually a charge, time and parity reversal of the corresponding $|n\rangle_{I I}$ e.g. $|\uparrow\rangle^{\prime}=|\downarrow\rangle$. We also have a choice, whether to map $|0\rangle_{\text {in }}$ to $\left\langle\left. 0\right|_{\text {out }}\right.$ or to $\left\langle\left.\uparrow \downarrow\right|_{\text {out }}\right.$. Then taking the hermitian conjugate of (3), we find

$$
\Psi=\sum_{m, n} C_{m n}^{*}\left|n^{\prime}\right\rangle_{\text {out }} \otimes\left\langle\left. m\right|_{\text {in }},\right.
$$

and hence $C^{\dagger}$ is just the S-matrix. The invariant tensor product states (14) are then the de Sitter-invariant building blocks of the S-matrix. For example, with $|0\rangle_{\text {in }} \rightarrow\left\langle\left.\uparrow \downarrow\right|_{\text {out }}\right.$, and with the rows and columns labeled in the order $\left.\mid 0\right\rangle,|\uparrow\rangle,|\downarrow\rangle$, $|\uparrow \downarrow\rangle$, a de Sitter-invariant S-matrix is

$$
S=\left(\begin{array}{cccc}
0 & 0 & 0 & a \\
0 & b & 0 & 0 \\
0 & 0 & b & 0 \\
c & 0 & 0 & 0
\end{array}\right),
$$

where we have used $T|\uparrow\rangle=\eta|\downarrow\rangle$ and $T|\downarrow\rangle=-\eta|\uparrow\rangle$ for time-reversal on spinors. If $a, b$, and $c$ are all phases, then the S-matrix is unitary as well as de Sitter-invariant.

We have shown by construction that a finite-dimensional Fock space is not in conflict with the de Sitter symmetries. To illustrate the point, we used a very small representation in which there were as few as four states. We mention, though, that by considering larger representations, one can see the emergence of geometric structure in the form of a fuzzy sphere at the boundary of de Sitter space 6 .

M. P. is supported by DOE grant DF-FCO2-94ER40818.

\section{References}

1. T. Banks, "Cosmological Breaking Of Supersymmetry, or Little Lambda Goes Back To The Future II," hep-th/0007146

2. W. Fischler, Talk at the 60th birthday of G. West, June 2000 .

3. N. Goheer, M. Kleban, and L. Susskind, "The Trouble with de Sitter Space," JHEP 0307 (2003) 056; hep-th/0212209

4. E. Witten, "Quantum Gravity in de Sitter Space," hep-ph/0106109

5. A. Guijosa and D. A. Lowe, "A New Twist on dS/CFT," hep-th/0312282

6. M. Parikh and E. Verlinde, to appear.

7. E. Schrödinger, "Expanding Universes," Cambridge University Press, 1956.

8. M. Parikh, I. Savonije, and E. Verlinde, "Elliptic de Sitter Space: dS/Z 2 ," Phys. Rev. D 67 (2003) 064005; hep-th/0209120

9. G. Gibbons, "The Elliptic Interpretation of Black Holes and Quantum Mechanics," Nucl. Phys. B271 (1986) 497.

10. G. 't Hooft, "On the Quantum Structure of a Black Hole," Nucl. Phys. B256 (1985) 727. 